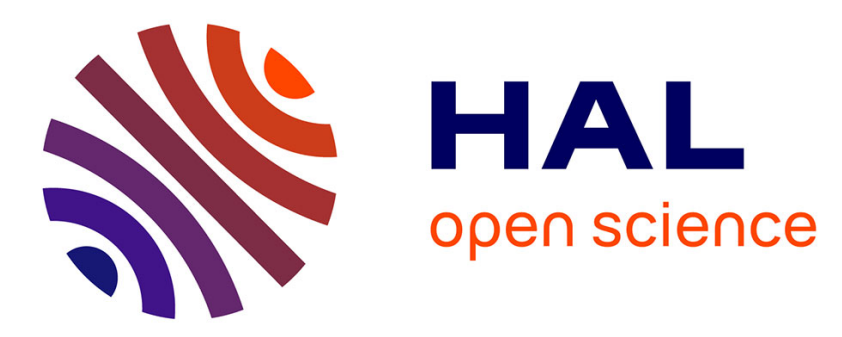

\title{
Pratiques syndicales, racisme et antiracisme. À propos d'une recherche en cours dans une entreprise de transports publics
}

Philippe Poutignat, Christian Rinaudo

\section{- To cite this version: \\ Philippe Poutignat, Christian Rinaudo. Pratiques syndicales, racisme et antiracisme. À propos d'une recherche en cours dans une entreprise de transports publics. Cahiers de la Méditerranée, 2000, 61, pp.273-287. halshs-00080728}

\section{HAL Id: halshs-00080728 \\ https://shs.hal.science/halshs-00080728}

Submitted on 20 Jun 2006

HAL is a multi-disciplinary open access archive for the deposit and dissemination of scientific research documents, whether they are published or not. The documents may come from teaching and research institutions in France or abroad, or from public or private research centers.
L'archive ouverte pluridisciplinaire HAL, est destinée au dépôt et à la diffusion de documents scientifiques de niveau recherche, publiés ou non, émanant des établissements d'enseignement et de recherche français ou étrangers, des laboratoires publics ou privés. 
COlloque Politique et Alterite : LA SOCIETE FRANÇAiSE FACE AU RACISME

Nice, 9,10,11 décembre 1999

Texte publié dans : Cahiers de la Méditerranée, n 61, décembre 2000, p. 273-287

Philippe Poutignat et Christian Rinaudo

(SOLIIS-URMIS, CNRS et Université de Nice-Sophia Antipolis)

\section{Pratiques syndicales, racisme et antiracisme. À propos d'une recherche en cours dans une entreprise de transports publics}

Nous présentons ici les premiers éléments d'une recherche en cours sur le " racisme au travail ", pour reprendre le titre du livre de Philippe Bataille (1997). Cette recherche, comme celle dont le livre précédemment cité est issu, s’inscrit dans le cadre d'une demande d'enquêtes et de réflexions portant sur le racisme et les discriminations dans les entreprises et dans la vie syndicale, adressées par de grandes confédérations, ici la CGT, à des chercheurs en sciences sociales. Précisons que la recherche dont nous allons rendre compte qui porte sur la société des transports urbains de Nice (et dans laquelle nous sommes plus particulièrement engagés), n’est qu'une des initiatives prises dans le cadre de ce dispositif de collaboration entre chercheurs et syndicalistes, lui-même inséré dans un programme européen ayant une dimension comparative ${ }^{1}$.

En dépit des limites de notre propos, nous rappellerons en préalable le contexte commun à ces recherches ainsi que des éléments de problématique qu'elles partagent. À cet égard, la critique de l'anti-racisme conjuratoire menée par Taguieff qui fait ressortir l'existence d'un nouveau racisme, intégré dans le nationalisme xénophobe, visant l'immigration et centré sur l'identité culturelle, plutôt que sur les catégories de race conformes au racialisme classique, peut sans doute être mise en regard avec cette prise de conscience des grandes centrales qu'il est insuffisant d'afficher son anti-racisme pour être à l'abri du développement de discours et de conduites racistes ou discriminatoires, y compris en leur sein.

De ce point de vue, également, le travail de Philippe Bataille est un précédent utile et on ne s'étonnera pas de croiser à nouveau plusieurs des thèmes qu'il a mis en relief et qui contribuent à élaborer les questions que posent les multiples figures du racisme contemporain, étant admis que celui-ci ne peut être rapporté à une "essence" unique sous-jacente à ses formes concrêtes (Balibar, 1992).

Le racisme et la xénophobie ne sont évidemment pas des phénomènes nouveaux dans le monde ouvrier, cependant leur place dans les relations de travail a changé en même temps que se renouvelait la thématique raciste et que la montée d'un discours politique xénophobe, et la politisation corrélative de ce qui dans le débat public est apparu comme "le problème de l’immigration ”, modifiait le contexte idéologique global. Cette politisation a permis une

1 Programme OSIME, “ Organisations syndicales, Immigrés et ninorités ethniques en Europe ”. Le volet français comprend outre plusieurs monographies d'entreprises ou d'établissements, des enquêtes sur les discriminations dans l'accès à l'embauche centrées sur des bassins d'emploi. Cf. “ Programme OSIME, Rapport d'étape 1997-1998”, Les Documents de I'ISERES, N07, Institut Syndical d'Etudes et de Recherches Economiques et Sociales, Montreuil, 1999. 
légitimation nouvelle à une représentation raciste de l'univers social, voire à un thème comme celui de la “préférence nationale" de trouver des relais dans le monde du travail. La cible même des expressions de racisme et de xénophobie se transformait ; la notion d'immigré a perdu sa relation avec la migration proprement dite, au profit d'une notion “d'origine ”, visant des catégories d'autres, essentiellement des populations issues de l'immigration postcoloniale, réputées inassimilables et dangereuses pour la société “ normale ”. Comme le relève Bataille2 , les acteurs syndicaux se sont trouvés démunis devant des manifestations de racisme qui ne coïncident plus avec la xénophobie ouvrière traditionnelle et que la dénonciation de l'exploitation patronale et l'appel à la solidarité ouvrière ne suffisent plus à contenir. Les représentations et stéréotypes véhiculés dans le champ politique donnent forme à un conflit identitaire larvé entre français d'origine (une catégorie elle-même ethnicisée) ou d'anciennes immigrations assimilées, et les immigrés plus récent d'Afrique du Nord ou d'Afrique Noire et leurs enfants. Ainsi la sphère du travail devient-elle, d'une façon qui lui est spécifique, le théâtre de la résistance à une intégration réussie de bon nombre d'enfants d’immigrés qui, outre la nationalité française, peuvent faire valoir des niveaux de compétence professionnelle équivalents à ceux de leurs camarades. Dans l'entreprise, l'intolérance à l'égard des manifestations de différence culturelle et religieuse se trouve justifiée par une non conformité au modèle d'intégration et aux règles de fonctionnement de la société, attribuée le plus souvent aux maghrébins. Cette intolérance s'adresse en fait à des jeunes imprégnés de culture urbaine dont on redoute qu'ils perturbent le cadre des identités au travail. Le monde ouvrier n'apparait plus, dès lors, comme le lieu par excellence d'intégration à une identité de travailleur, mais plutôt comme un champ potentiel de conflits trouvant à se formuler en termes identitaires et culturels, confirmant et aggravant la perception, déjà bien établie, d'un affaiblissement du principe d'unité de la classe ouvrière.

La conclusion la plus importante que l'on peut tirer de ces travaux réside dans un double constat : la banalisation du racisme a pour conséquence qu'il est à la fois très présent dans les ambiances de travail et peu visible dans les mécanismes structurels ou systémiques par lesquels s'effectuent la discrimination dans le travail (la distribution des tâches, leur ethnicisation, l'avancement des carrières) et à l'embauche. Cette dernière, par exemple, peut être orchestrée, en dehors de tout plan délibéré, par une pluralité d'acteurs partageant des évidences susceptibles de rester tacites. Elle met ainsi en jeu une combinaison de critères de stigmatisation: appartenance sociale (une identité de pauvre, on dira de "défavorisé "), territoriale (" quartiers difficiles”), ethnique ou culturelle (“d'origine immigrée ”), de couleur..., pour constituer une figure ou un type social racialisé, ou plus exactement racialisable, tel celui du " jeune des banlieues ", sans pour autant que la marque raciale puisse s'autonomiser et se réduire à une identité raciale explicite " pure et simple " (comme "Noir " aux États-Unis).

Nous allons voir que ces types “ d'évidences” sont en effet constitutifs de la racialisation des relations sociales au-delà des cas de discrimination à l'embauche. Mais nous mentionnerons encore un autre cas de figure inventorié par Bataille et qui va nous concerner au premier chef : celui qui caractérise les services publics ou parapublics, dans la catégorie desquels se situent les transports urbains. Prolongeant des analyses proposées par Wieviorka (1991), il montre que s’agissant d'emplois institutionnellement protégés par la "préférence nationale ", les manifestations du racisme se traduisent principalement dans le rapport aux usagers ou aux clients. Celui-ci est vécu sur le mode d'un dysfonctionnement à l'égard duquel la figure de l'autre sert de repoussoir : il incarne une source de désordre au coeur d'un sentiment de crise professionnelle tenue elle-même pour la manifestation d'une crise générale

2 voir également Christian Poirret (1997) 
des valeurs, de l'autorité, de la moralité et, en définitive, d'une remise en cause de l'identité nationale.

C'est donc à une variante de ce cas de figure que nous sommes confrontés. Comme nous l'avons dit, beaucoup des thèmes évoqués jusqu'ici concernent notre recherche, mais notre démarche descriptive, nous amène à les traiter autrement. Nous laisserons en suspens la question de la place qu'occupent les manifestations contemporaine du racisme dans un tableau d'ensemble de changements macro-sociaux susceptibles de les affecter ("la fin de la société industrielle ”, “la crise des institutions ”, “ la poussée des identités culturelles ”)3 et, par là même, celle du sens qu'on leur attribue. Autrement dit, en étudiant une situation particulière, nous ne cherchons pas à illustrer un cadre interprétatif global indexé à une période historique (qui se trouverait être la nôtre), définissant des catégories d'acteurs et des logiques d'action qu'il s'agirait alors de documenter et de détailler. Dans ce colloque où dominent légitimement des travaux d'historiens, nous ne pouvons faire autrement que d'avouer un objectif sociologique modeste : tenter de restituer des dynamiques locales qui établissent les rapports entre des " nous" et des " eux " essentialisés. Leur compréhension, loin d'être réductible à des significations stipulées d'emblée, suppose la prise en compte de pertinences déployées dans des champs d'interlocutions et d'interactions qu'il s'agit justement de décrire. Cette démarche vise donc à rendre compte de définitions de situations qui, du point de vue des acteurs constituent les cadres à la fois cognitifs et moraux dans lesquels ils agissent. Elle se propose également de faire des hypothèses sur les évolutions possibles de consensus locaux et les modifications de ces cadres. L'accent mis sur les définitions de situations, rapportables aux acteurs, à leurs discours et à leurs pertinences pragmatiques, n’implique nullement de négliger des dimensions contextuelles globales transcendantes aux situations ; elle oblige cependant à les trouver, ou à les retrouver, comme configurations de sens convoquées et soutenues localement, dans l'actualité des relations sociales.

Ainsi, une des configurations de sens pertinentes dans la situation étudiée concernera cette nouvelle figure de l'altérité que sont les “ jeunes des banlieues ”. Cette dernière s’est imposée avec force ces dernières années, à côté d'autres figures comme celles des “clandestins ” et des “sans papiers”. Comme nul ne l'ignore, puisqu'il s'agit là d'une nouvelle doxa, sa caractéristique consiste à ne pas représenter n'importe quel jeune et n’importe quelle banlieue, mais les “jeunes issus des quartiers défavorisés”. Quant à la configuration qui contribue à lui donner son sens, elle est centrée sur la notion d'insécurité, (dont différentes déclinaisons peuvent impliquer également “les clandestins" et les “sans papiers"), celle qui nous intéresse directement, renvoyant au problème dit de “l'insécurité urbaine ”. Celle-ci à fait l'objet depuis quelques années, de toutes sortes de tentatives d'objectivation couplées avec les mesures de politiques de la ville4. Soulignons que cette problématique (sociale) conduit à mettre en relation l'immigration, non plus avec des questions de concurrence sur le marché du travail (les immigrés qui prenne le pain des Français), mais avec l'identification d'un ennemi, - proche mais pourtant moralement, culturellement, civilement distant - le fauteur de trouble. Le “ jeune des quartiers”, ainsi entendu, a d'ailleurs reçu sa dénomination quasi-officielle de “ sauvageon ”. Celle-ci vient connoter une autre catégorie, celle “d’incivilités ”, servant à lier dans l'entendement bureaucratique des phénomènes de violence phénoménalement hétérogènes. Cette notion, à laquelle on peut trouver des lettres de noblesse dans la théorie

\footnotetext{
3 Cf. M. Wievorka (1998), chap.5.
}

4 Une de ces objectivations consiste par exemple dans la construction d'une sorte “ d'échelle de Richter ” de la violence urbaine construite par les renseignements généraux, qui a reçu une certaine publicité médiatique et a été souvent reprise dans des rapports officiels. Cf. V. Laurent (1999) 
sociologique et la théorie politique, désigne des actes déviants et des “petites violences" quotidiennes qui ne tombent pas directement sous le coup de la loi et auquel on attribue un " sentiment d'insécurité " traité lui-même comme un objet en soi5 ; celui-ci est invoqué, en retour, pour attester, comme d'un fait sensible, de la continuité6 entre atteintes à la " civilité " (sous le même paradigme que " savoir-vivre ”, “politesse ”, “ tact ”...) et atteintes à la paix civile, comme forme de vie propre au gouvernement politique, opposé à l'état de nature.

Dans cette configuration qui lie question urbaine, question de l'insécurité et contribue à reposer sous une nouvelle forme une " question de l'immigration ”, les métiers des transports urbains sont l'un des symboles d'une mise en crise du lien civil par la violence (ce n'est évidemment pas le seul, pensons au problème dit de “la violence à l'école ”) inscrite aujourd'hui comme l'une des principales questions de société dont L. Jospin affirmait récemment dans Le Monde qu'elle est l'une des principales préoccupations de son gouvernement.

Nous nous sommes donc proposé d'inventorier et de décrire comment, dans ce contexte, les conditions propres à l'entreprise, les situations de travail caractéristiques des transports urbains, et les évolutions qu'elles connaissent, font place au racisme, et, corrélativement, aux actions susceptibles de s'y opposer7,

Nous commencerons par présenter la société de transport que nous étudions.

Les transports urbains de Nice sont placés, comme la plupart des réseaux en France, sous l'autorité organisatrice de la municipalité. Celle-ci désigne ensuite un concessionnaire qui reçoit une enveloppe globale destinée à la prise en charge du réseau et qui sous-traite les travaux de mise en œuvre à un opérateur de transport, une société privée qui appartient pour $50 \%$ à la CGEA (groupe Vivendi) et pour 50\% à GTI (groupe VIA Assurances).

Cette entreprise compte environ six cent cinquante employés dont cinq cents conducteurs receveurs, quatre-vingt cinq ouvriers d'entretien (mécaniciens, électriciens, service de nettoyage) et une vingtaine d'agents de maîtrise. Une de ses particularités vient de l'importance que représente la CGT dans les différentes instances représentatives : conseil de discipline, comité d'entreprise... Aux dernières élections professionnelles, le syndicat CGT des transports urbains de Nice obtenait $86,9 \%$ des suffrages contre 10,9\% pour la CFDT et $2,2 \%$ pour Force ouvrière. Couplé à un taux de syndicalisation qui est resté très important comparé à d'autres secteurs professionnels (environ 75\% des salariés de l'entreprise), cette large concentration des forces syndicales dans les mains de la CGT a amené ses responsables

5 Aussi, selon la doxa, faudrait-il dire plutôt “le sentiment d'insécurité ”. On en trouvera un remarquable exemple dans un tout petit livre dont l'objet, qui peut se résumer par la formule “ en peu de pages, tout ce qu'il faut savoir sur les violences urbaines”, est quasi explicitement doxique. C. Soullez (1999), Cf. notamment pp 67, “Le sentiment d’insécurité ” et passim..

6 postulable en théorie, du moins sous la forme positive d'une continuité entre sociabilité quotidienne, avec ses contraintes d'intelligibilité mutuelle, et ordre politique, avec ses contraintes de légitimité, Cf. par exemple P. Pharo (1992)

7 ce qui, bien évidemment, présuppose des acteurs sociaux, syndicaux en premier lieu, engagés dans une démarche réflexive anti-raciste qui va bien au delà de la simple dénonciation rituelle. Au stade actuel de la recherche, la présentation faîte ici reste entièrement dépendante des limitations et faiblesses d'analyse propre aux chercheurs, mais elle n'aurait tout simplement pas pu exister sans que cet engagement se soit d'ores et déjà activement manifesté. 
à entrer dans une dynamique de cogestion de l'entreprise en ce qui concerne tous les services directement liés à l'exploitation du réseau. Une telle logique a engagé ce syndicat dans la mise en œuvre d'une politique mesurée, prenant quelquefois ses distances avec les revendications de sa base, tout en se montrant très présent dans le domaine de l'amélioration des conditions de travail, mais également et plus généralement, grâce à son implication au sein du comité d'entreprise, dans ce qui touche à l'organisation des activités para et extraprofessionnelles (restauration d'entreprise, activités sportives et culturelles, prise en charge des enfants du personnel lors des vacances scolaires, etc.). Ainsi définie, la ligne syndicale de cette organisation peut se résumer par sa volonté de concilier son engagement dans la bonne marche de l'entreprise (ce qui implique, contrairement aux revendications des salariés, de ne pas arrêter systématiquement le travail à chaque fois qu'un incident se produit sur le réseau ${ }^{8}$ ) tout en exerçant un fort pouvoir syndical vis-à-vis de la direction: menace de grève, démonstration de force comme lorsque les trois cents bus du réseau se sont retrouvés, par un simple appel radio d'un responsable syndical, devant la préfecture des Alpes-Maritimes à la suite de l'agression d'un chauffeur.

Une autre particularité de cette entreprise est qu'elle est décrite par l'ensemble des salariés comme “ une grande famille”, un peu comme l'était la “ famille cheminote” dont parlent Dubar et Tripier (1998), ce qui se traduit notamment par un sentiment partagé de forte solidarité interne qui dépasse les clivages catégoriels et idéologiques. Cette dimension familiale n'est d'ailleurs qu'en partie métaphorique dans la mesure où on peut effectivement observer une grande multiplicité des liens réels de parenté entre les salariés, certains étant présents dans l'entreprise depuis plusieurs générations. Il s'est ainsi développé une sorte de droit d'accès privilégié à l'entreprise pour les membres de la famille des salariés, favorisé par la direction, et largement relayé par le syndicat qui, face à la crise du marché de l'emploi, s'est vu dans l'obligation de prendre en compte cette revendication formulée par sa base. Ce type d'accord contribue au développement d'un "corporatisme contractuel" (Dubar et Tripier), fondé sur la négociation entre l'employeur et le syndicat, et qui se concrétise par un accord tacite pour embaucher un minimum de 50\% de fils d'employés. "Si on descend en dessous des 50\%, explique un responsable syndical, on les plante et ils sauront pourquoi on le fait ”).

Cette représentation de la “grande famille” se traduit également par l'adhésion du personnel à des normes de comportement “ maison ” entretenues par des rituels de politesse impératifs entre les membres qui se manifestent une considération mutuelle (serrements de mains à tout le monde), par une virilité ostentatoire et, de façon corrélative, par une " rugosité " des manières de parler dont la signification pour l'identité professionnelle est soulignée par un lien revendiqué aux ancêtres charretiers. Une conséquence en est aussi que les termes dérogatoires pouvant servir d'injure raciste (“melons”, “ gris”) sont en usage constant dans l'entreprise, et trouvent à se prévaloir de ces façons habituelles et valorisées : celles, notamment, d’un usage viril et non euphémisé du langage que résume bien la formule consistant à prévenir les nouveaux venus et, en premier lieu, les universitaires qui

\footnotetext{
8 Voici comment, à la suite de l'agression d'un chauffeur, le syndicat justifie dans un tract distribué aux salariés son refus d'entrer dans une logique systématique d'arrêt du travail : “ Il est certain que, pour s'en tenir aux agressions, aux menaces avec arme, nous aurions dû arrêter le travail 18 fois depuis le début de l’année. Si l'on rajoute les jets de gaz et les crachats, c'est 37 fois de plus, soit 55 arrêts de travail en dix mois qu'il aurait fallu faire. [...] Cela ne veut pas dire que nous ne ferons plus grève pour la sécurité. Nous en referons sûrement, mais à bon escient, pour obtenir des mesures supplémentaires, mais en sachant que nous vivrons avec cette violence urbaine malheureusement encore longtemps. ”
} 
s’intéressent à la question du racisme dans le monde du travail, que “ ici, on appelle un chat un chat".

Ajoutons que l'on peut trouver dans l'entreprise un usage habituel des catégorisations raciales qui cependant ne la particularise sans doute pas. Il témoigne plutôt de préconstruits discursifs et de représentations sociales qui, comme le souligne Taguieff, reconduisent le présupposé de l'existence des races lequel fait ainsi partie d'un stock d'évidences communes en-deça des clivages entre racistes et antiracistes. On les retrouve d'ailleurs aussi bien chez certains des employés susceptibles d'être en butte aux préjugés de leurs collègues.

Cet usage des catégories est également au fondement de la racialisation d'une géographie sociale qui contribue à une connaissance de sens commun de la structure sociale pas différente de celle qui met en oeuvre les catégories, apparemment plus éduquées et plus savantes, de “ quartiers difficiles”, ou de “ ghettos" (Rinaudo, 1999). Cette géographie de sens commun s'élabore en mobilisant des catégories et des finalités pratiques, qui consistent notamment à établir quelles sont les lignes difficiles, celles qu'on évite et qui sont traditionnellement attribuées aux nouveaux chauffeurs. Elles sont intimement liées à la question de l'insécurité telle que ces derniers la rencontrent dans leur pratique professionnelle.

Dans les plaintes des chauffeurs, la question de l'insécurité est directement associée à des considérations sur la perte de prestige de la profession et se traduit souvent par le sentiment partagé de ne plus être respectés. Toutefois, ils différencient nettement ce qui relève d'une perte de considération générale des usagers à leur égard, de ce qui découle d'actes assignables à une catégorie particulière avec laquelle ils sont, par conséquent, dans une relation conflictuelle. Dans le premier cas, la plainte s’adresse aux usagers en général qui manifestent régulièrement de l'indifférence à leur égard ${ }^{9}$, voire certaines formes d'hostilité, en cas de retard par exemple, mais alors cela ne leur est pas directement destiné, mais à travers eux, “ aux bus ”. L'absence de considération fait d'eux une sorte de “ machine à conduire10 ”, et va de pair avec une sorte d'invisibilisation de leur personne ${ }^{11}$. Dans l'autre cas, tout au contraire, le manque d'égard dont ils se sentent l'objet les met sur le devant de la scène. Il va de pair avec une forte visibilité et concerne les incivilités, c'est-à-dire les comportements qui dérogent non seulement à ce que l'on peut attendre d'un usager “ normal ”, mais également aux attentes des usagers “normaux” eux-mêmes. Ici sont évoqués non seulement des comportements directement agressifs à leur égard (insultes, crachats), mais aussi des comportements tels que la fraude, le chahut, le fait de fumer et, d'une façon générale, le refus d’obtempérer. Ceux-ci se déroulent sous les yeux des autres passagers qui sont alors fondés à se plaindre. Ils remettent en cause, pour le chauffeur, son statut de maître à bord, attentif à ce qui se passe dans son bus. Or l'une des contraintes de la profession consiste, compte tenu du caractère nécessairement public des conduites, en une forte exigence de justifiabilité. Ainsi, par exemple, ne pas intervenir dans des cas de fraudes manifestes, c'est être sous la menace de se voir reprocher de ne pas accorder le même traitement à tout le monde, reproche

\footnotetext{
9 Un chauffeur : “ Les gens ne disent pas bonjour, ils ne disent pas merci, ils ne disent pas s’il vous plaît... Souvent ils vous mettent un billet de cent francs dans les mains et ne vous demandent pas ce qu'ils veulent. À vous de le deviner. En règle générale il n’y a pas de contact, les gens ne vous disent pas bonjours, ils ne sont pas polis, si vous ne faites pas comme ils veulent, ils vous insultent..."

10 Un chauffeur: “Tu fais partie du mobilier. Les gens ne voient pas quand ils rentrent qu'il y a un être humain qui est en train de conduire un bus... Ils montent dans un bus, ils payent. Ils nous prennent pour des robots."

11 Un chauffeur : “Quand les gens vous demandent pour aller quelque part, c’est : “il va où, le bus ?” Il va où je le mène... “Et le chauffeur, il va où ?” Donc quand vous entendez ça, vous avez tout compris.
} 
particulièrement difficile à supporter parce qu'il contrevient à l'éthique égalitaire de la profession. Dès lors, ces comportements sont attribués à “ceux qui nous emmerdent” et ceux-là sont, dans le langage de l'entreprise, ceux qui nous empêchent d'exercer notre métier et de manifester les qualités professionnelles qui vont avec, notamment sur les "lignes difficiles . "Ceux qui nous emmerdent" constitue ainsi une autre manière de désigner ces “jeunes des quartiers”, (ce qui peut également se résumer par cette formule, conforme aux expressions d’usage : “ ces petits melons qui nous emmerdent ”).

Ainsi la question du racisme dans ce milieu professionnel porte moins sur la division des travailleurs en fonction de leur origine nationale que sur la désignation d'un " ennemi ", extérieur à l'entreprise, et nettement localisé et identifié. Cette position du problème se retrouve dans cette déclaration d'un délégué du personnel lors d'une réunion confédérale consacrée, précisément, à la question des discriminations raciales au travail : “ Dire qu'on a des problèmes racistes dans notre entreprise, ça serait vrai et faux. Le problème qu'on a vient de l'extérieur et les collègues qui sont Maghrébins et qui travaillent dans notre entreprise en subissent les conséquences, indirectement malheureusement... Ça se passe par rapport aux bandes de jeunes qui, dans les quartiers défavorisés, se regroupent avec pour premier but de se venger de l'État qui les laisse choir dans les cités.”

Cette déclaration souligne que le racisme, en tant qu'il concernerait directement les relations de travail, n'est pas à considérer comme un problème central dans l'entreprise, mais comme un effet indirect du ressentiment des chauffeurs à l'égard de ce qu'ils ont à subir de la part de ces jeunes qui fréquentent les “ lignes difficiles” du réseau. Elle nous permet ainsi d'introduire la question du traitement du racisme par le syndicat.

En premier lieu, notons que les responsables syndicaux font explicitement la différence entre les salariés qui ont des réactions qui peuvent être qualifiées de racistes, et les racistes proprement dits. Ceux-là sont connus et bien identifiés par leurs idées “ extrémistes ”, c’est-àdire par leurs positions politiques et idéologiques d'extrême droite. Cette caractérisation en fait des acteurs ultra-minoritaires, et, selon la mémoire historique transmise par les plus anciens, les restes défaits d'une bataille gagnée depuis longtemps contre les tentatives d'implanter des syndicats patronaux tels que la CFT dans les années soixante-dix, avec le concours d'éléments rapatriés d'Algérie plus ou moins compromis avec L’OAS. Il demeure cependant toujours le risque que certains, dans ce contexte, se laissent séduire par des idées extrémistes dont l'incarnation est aujourd'hui le Front National, ou deviennent obsédés par leur ressentiment ou leur haine à l'égard des immigrés. Ceux-là sont alors considérés comme des “ cons" et la pédagogie syndicale consiste à faire la démonstration qu'une telle obsession rend le travail encore plus difficile et, d'autre part, qu'elle ne consiste en fait qu'en rodomontades de faux “ durs ”, incapables de traiter réellement le problème de l’insécurité.

Quelles sont, dans ce contexte, les réponses apportées par l'organisation syndicale ? Elles consistent essentiellement à tenter de "calmer le jeu" et de contenir ces formes de manifestations racistes afin qu'elles ne dégénèrent pas en obsessions anti-immigrées. Cela implique de faire la part des réactions que l'on juge passagères et liées à un surcroît de tensions, comme par exemple, en laissant les chauffeurs se “défouler" dans les lieux de coulisse (lors des prises et des fins de service au dépôt, au snack d'entreprise...) de manière à libérer leur haine accumulée pendant leur journée de travail, quitte à ce que les collègues d'origine maghrébine les entendent et risquent de le prendre pour eux, alors même que les propos racistes proférés à ces occasions ne s'adressent pas directement à eux, comme le laissait entendre la déclaration précitée.

Dans sa dynamique de “ cogestion” de l'entreprise, le syndicat, comme la direction, a cherché, dès le début des années quatre-vingt-dix, à apporter des solutions concrètes aux 
problèmes de sécurité dans les transports urbains, que ce soit dans son acception générale (sécurité routière...) ou dans sa formulation plus directement centrée sur les agressions du personnel et sur les actes d'incivilité qui perturbent et mettent en crise l'ensemble de la profession. Loin d'être isolées ou particulièrement originales, les initiatives locales en matière de lutte contre l'insécurité s'inscrivent dans un contexte général de prise en compte de ce problème et de mise en œuvre de mesures visant à sa résolution. Cependant le syndicat a proposé plusieurs de ces mesures et a milité activement pour que soit établi dans le contrat local de sécurité, une convention “pour la sécurité dans les transports urbains ”. Un certain nombre d'initiatives ont déjà été prises, comme l'instauration d'un d'observatoire de l'insécurité permettant une comptabilisation des incidents survenus sur le réseau, la création d'une brigade d'intervention ${ }^{12}$ et, plus récemment, le recrutement d'une vingtaine d'agents locaux de médiation sociale qui ont pour mission d'accompagner les chauffeurs dans les bus. Si l'on retrouve toutes ces initiatives dans la plupart des grands réseaux urbains, une différence notable est peut-être qu'ici le syndicat CGT a pu se présenter comme l'un des principaux acteurs de la définition du problème de l'insécurité et de la mise en œuvre de ces mesures.

Une des manières de “calmer le jeu” a alors été de favoriser la création d’une brigade d'intervention mobile (BMI) et d'en contrôler la définition des tâches et l'organisation de ses services. Il s'agit de trois équipages qui interviennent à tout moment sur le réseau à la fois pour des missions d'assistance aux chauffeurs destinées à dégager les couloirs de circulation et les arrêts de bus, et sur des actions de prévention et de médiation sociale qui consistent essentiellement à négocier des arrangements avec les "jeunes des quartiers" de manière à maintenir des apparences normales tout en leur accordant de facto un statut d'usagers particuliers. Par exemple, une des interventions couramment effectuées consiste à se placer à des arrêts stratégiques et à laisser monter sans payer les jeunes identifiés comme de potentiels fauteurs de troubles en leur demandant de se tenir tranquille tout au long de leur trajet. Une autre de leurs missions les amène à se rendre périodiquement dans les quartiers périphériques desservis par les lignes “ difficiles” pour se faire connaître personnellement des jeunes les plus visibles dans les lieux publics et prendre des contacts avec les responsables des associations les plus influentes.

Or, cette stratégie de médiation se heurte à la volonté quasi générale des chauffeurs d'appliquer un principe d'égalité consistant, par des mesures de répression plus sévères, à obliger les faiseurs d'insécurité à se comporter comme des usagers “ normaux ”. En voulant “ calmer le jeu " et contenir les manifestations du racisme par un travail de médiation sociale, le syndicat va à l'encontre du principe de justice valant pour tous défendu par les chauffeurs. Plus encore, cette manière de favoriser le contact social avec les membres du groupe identifié comme marginal est toujours susceptible de renforcer chez certains chauffeurs ce qu'Élias et Scotson ont appelé la “ peur de la pollution ". Les jeunes en question étant réputés anomiques, frayer avec eux, c'est courir le risque d'une “infection anomique”, c'est donc être soupçonnable de briser les normes et les tabous de son groupe (1997, p. 39). C'est ainsi que les membres de cette brigade sont systématiquement accusés, selon la formule d'usage, d'aller “ distribuer des bonbons aux melons de l'Ariane ”. Les laisser monter dans le bus sans payer sous le regard des autres usagers, aller discuter avec eux au fond du bus au lieu de leur dresser des procès verbaux revient, dans le langage imagé de l'entreprise, à “ baisser le pantalon ”.

12 Il s’agit de trois équipages destinés à intervenir à la fois sur des missions d'assistance aux chauffeurs (dégager les couloirs de circulation et les arrêts de bus...) et sur des missions de médiation sociale avec les jeunes qui sont à l'origine des troubles repérés par l'observatoire des incidents 
Une autre manière de prendre en charge le problème de l'insécurité en y apportant des réponses concrètes revient à instaurer un corps spécialisé de gestion des relations interethniques en ethnicisant les tâches en fonction de compétences spécifiques liées aux origines ethniques et socio-urbaines des intervenants. C'est ce qui a été amorcé lorsqu'un jeune d'origine tunisienne, issu d'un quartier défavorisé de la ville, a été intégré dans la brigade.

Pour les membres de la brigade, cette nouvelle recrue a très vite été considérée comme un agent "hors pair", ayant de grandes qualités professionnelles, notamment du fait de ses compétences qu'il tenait d'être lui-même originaire d'un “ quartier ” et qui consistaient en des savoir-faire sur la manière d'aborder les relations avec les jeunes à l'origine des troubles et sur la capacité de décision quant à la manière d'agir dans les situations les plus délicates. De telles compétences se traduisent notamment par sa manière de distinguer à partir d'indices imperceptibles pour les non initiés les “ cons ” de la " racaille ” : "Il réfléchissait comme eux, explique un membre de l'équipe, avec un raisonnement que nous, on ne peut pas avoir. Ça, je l'ai compris en travaillant avec lui. Pour lui, [ces jeunes en question] ce sont des cons jusqu'à un certain point... Après, c'est de la racaille (...) Je lui disais : "Mais pour moi, ce sont les mêmes...” Et non, ce ne sont pas les mêmes! ” Autrement dit, c'est parce qu'il est “ comme eux " que ce jeune peut raisonner de cette manière et manipuler ces catégories de la pratique qui sortent de l'entendement des gens “ comme nous ”. Or, c'est bien à partir de la maîtrise de ces catégories (qui est con? qui est une racaille ?) que se déterminent les choix d'action dans les situations où l'intervention de la brigade est jugée nécessaire, et notamment les décisions de négocier ou de se montrer intransigeant, de faire descendre le contrevenant ou de le raisonner, etc.

Outre le fait que l'intégration de ce jeune d'origine tunisienne dans la brigade est perçue comme un acte de traîtrise supplémentaire par ceux qui dénonçaient déjà les stratégies de médiation — le chef d'équipe est alors désigné comme “ celui qui embauche les Arabes ”-, on peut voir dans la valorisation de cette initiative un durcissement de l'ethnicisation des relations sociales qui peut apparaître comme une contradiction avec les objectifs initiaux du syndicat. Celui-ci se trouve enfermé dans une logique paradoxale où il en vient, pour contenir les comportements racistes qui s'expriment dans l'entreprise à l'encontre de ces jeunes “ des quartiers ", à favoriser une ethnicisation des tâches en fonction de compétences et de savoirfaire attribués sur une base ethnico-culturelle.

Aujourd'hui, ces contradictions se posent avec une acuité particulière dans l'entreprise du fait du recrutement récent d'une vingtaine et bientôt d'une quarantaine d'agents de médiation qui ont pour mission d'accompagner les chauffeurs dans les bus. Dans un premier temps, le syndicat s'est fermement opposé à l'idée même de recrutements d'emplois de types “ grands frères " ou " agents de médiation ” en combinant un double refus, celui des emplois précaires que supposait l'embauche de jeunes dans le cadre de mesures incitatives favorisées par la politique de la ville et celui des critères ethniques sous-jacents à la notion de " grand frère ". La décision prise d'accepter leur embauche a finalement été justifiée par les meilleures conditions salariales et surtout par les possibilités de formation et à terme d'intégration dans l'entreprise que garantissait le nouveau statut d'emploi jeune. Elle a également été conditionnée par le recrutement d'un quota de 50\% d'enfants du personnel.

Au total, ce fut donc 24 jeunes qui furent retenus dont la moitié sont des enfants du personnel. Un des effets de ce quota a été que l'autre moitié a été recrutée, de fait, selon la logique implicite à l'œuvre avec la notion d'agents de médiation que renforce encore le contraste avec les $50 \%$ d'enfants du personnel. On peut constater en effet qu'en pratique, dans la constitution des équipes que l'on rencontre aujourd'hui dans les bus de la ville, s'est instauré une "ligne de couleur”. En effet, les binômes ont été constitués de telle manière 
qu'ils combinent à chaque fois des critères de mixité sexuelle - de manière à ce que les garçons puissent venir en aide aux filles en cas de nécessité — à des critères de mixité raciale — un Blanc avec un Maghrébin ou un Noir —, le tout étant volontairement combiné de manière à ce que ceux qui correspondent le mieux à la figure prototypique du jeune de banlieue se retrouvent systématiquement avec des enfants du personnel pour anticiper d'éventuelles réactions hostiles des chauffeurs.

Ainsi, la situation telle qu'elle se présente aujourd'hui manifeste une contradiction entre deux logiques à l'œuvre dans l'action syndicale. La première, conforme aux orientations profondément enracinées dans le mouvement syndical, conjugue le refus des propositions patronales susceptibles de créer des catégories d'emplois hors statut ou précaires avec le refus de la spécialisation ethnique de ces emplois et vise à leur intégration dans la structure professionnelle de l'entreprise. La seconde, celle qui correspond à l'intention de “ calmer le jeu ”, fait néanmoins une part à l'ethnicisation des tâches et risque à terme d'aller dans le sens d'un développement d'empois spécifiques liés à la sécurité. Comme nous l'avons évoqué, la prise en compte de la sécurité est une tendance lourde dans la redéfinition des missions du service public de transport. Ces deux logiques se sont trouvées combinées et, d'une certaine manière, équilibrées, dans le recrutement des emplois jeunes selon une ligne de partage 50/50. Mais un tel équilibre risque bien d'être instable et de nécessiter un renouvellement de la réflexion syndicale sur le racisme et l'ethnicisation dans l'entreprise.

Références bibliographiques

Balibar, E., Racisme, nationalisme, Etat. in, Les frontières de la Démocratie,Paris,Éditions La Découverte,1992.

Bataille, P., Le racisme au travail, Paris, Éditions La Découverte, 1997.

Dubar, C. et Tripier, P., Sociologie des professions, Paris, Armand Colin, 1998.

Élias, N. et Scotson J.L., Logiques de l'exclusion. Enquête sociologique au cœur des problèmes d'une communauté, Paris, Fayard, 1997.

Laurent, V. “Les renseignements généraux à la découverte des quartiers ”, Le monde Diplomatique, Avril 1999

Pharo, P. Phénoménologie du lien civil, Paris, L’Harmattan, 1992

Poiret, C., Discriminations ethniques et raciales : de quoi parlons nous?, in, Actes du Colloque “ le racisme et les discriminations au travail, Paris, ISERES, 1997

Rinaudo C., L'ethnicité dans la cité. Jeux et enjeux de la catégorisation ethnique, Paris, L'Harmattan, 1999.

Soullez, C. les violences urbaines, Paris, Les Essentiels Milan, 1999

Taghieff, P.-A, Le racisme, Paris, Flammarion, 1997.

Taghieff, P.-A., La force du préjugé. Essai sur le racisme et ses doubles, Paris, Gallimard, 1990.

Wieviorka, M., La démocratie à l'épreuve. Nationalisme, populisme, ethnicité, Paris, La découverte, 1993.

Wieviorka, M., Le racisme, une introduction, Paris, la Découverte/Poche, 1998. 